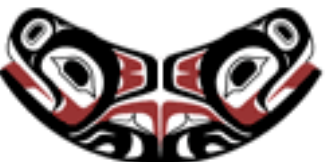

UW Biostatistics Working Paper Series

9-7-2006

\title{
Assessing the Adequacy of Variance Function in Heteroscedastic Regression Models
}

Lan Wang

University of Minnesota, lan@stat.umn.edu

Xiao-Hua Andrew Zhou

Department of Biostatistics, University of Washington, azhou@u.washington.edu

\section{Suggested Citation}

Wang, Lan and Zhou, Xiao-Hua Andrew, "Assessing the Adequacy of Variance Function in Heteroscedastic Regression Models" (September 2006). UW Biostatistics Working Paper Series. Working Paper 299.

http://biostats.bepress.com/uwbiostat/paper299

This working paper is hosted by The Berkeley Electronic Press (bepress) and may not be commercially reproduced without the permission of the copyright holder.

Copyright (c) 2011 by the authors 


\title{
Assessing the Adequacy of Variance Function in Heteroscedastic Regression Models
}

\author{
Lan Wang* \\ School of Statistics, University of Minnesota, 224 Church Street SE, \\ Minneapolis, MN 55455, U.S.A. \\ and \\ Xiao-Hua Zhou ${ }^{\dagger}$ \\ HSR\&D, VA Puget Sound Health Care System, 1100 Olive Way, 1400, \\ Seattle, WA 98101, U.S.A. \\ and \\ Department of Biostatistics, University of Washington, \\ F600, HSB, Box \# 357232, Seattle, WA 98198, U.S.A.
}

Summary. Heteroscedastic data arise in many applications. In a heteroscedastic regression model, the variance is often taken as a parametric function of the covariate or the regression mean. This paper presents a kernel-smoothing based nonparametric test for checking the adequacy of such a postulated variance structure. The test does not need to specify a parametric distribution for the random errors. It has an asymptotical normal distribution under the null hypothesis and is powerful against a large class

*email: lan@stat.umn.edu

† email: azhou@u.washington.edu 
of alternatives. Numerical simulations and an illustrative example are provided.

KEY WORDS: goodness-of-fit test, heteroscedastic errors, kernel smoothing, pseudo-likelihood, variance function 


\section{Introduction}

The problem of modeling heteroscedasticity frequently appears in practical data analysis. It is well known that modeling variance function is important for the efficiency of estimating the mean; the variance function itself may be of practical importance; and whether variance is appropriately taken into account could influence the estimation of other quantities of interest, such as confidence interval, prediction interval, test statistics. For example, in assay data analysis, the quality of estimation has been found to highly depend on the modeling of the variance structure (Davidian, Carroll and Smith, 1988). Such a data example (from Carroll and Ruppert, Section 2.8, 1988) is given in Section 5, which consists of 108 measurements from a calibration experiment of an assay for estimating the concentration of an enzyme esterase. The response variable $Y$ is the radioimmunoassay (RIA) counts, and the covariate $x$ is the concentration of esterase. A scatter plot of this data is given in the top panel of Figure 1.

[Figure 1 about here.]

The heteroscedasticity exhibited in this data set is evident. Larger variance is associated with larger response. This might encourage the researchers to consider a variance function that is a function of the mean, such as a powerof-the-mean variance model. Would this provide an adequate fit? Since the responses are counts, would a Poisson model be appropriate? See Ruppert et al. (1997), Zhou, Stroupe and Tierney (2001) for examples of heteroscedastic data in other areas.

To answer the above questions, we need to develop goodness-of-fit testing procedures for checking the adequacy of the variance function. Rigorous 
procedures for such a purpose are very lacking. Although many tests have been proposed for checking whether a variance function is constant or not, such as Breusch and Pagan (1980), White (1980), Cook and Weisberg (1983), Müller and Zhao (1995), Diblasi and Bowman (1997), Cai, Hurvich and Tsai (1998), these do not tell whether a specific variance function can adequately describe the variability in the data. Classical tests, such as the Wald test, the likelihood ratio test and the score test, may be constructed for this purpose but they require the specification of a specific alternative model and a parametric error distribution. Although the classical tests are powerful against that specified alternative, they may completely lose the power if the true alternative is not in the specified direction. Recently, Bedrick (2000) and Arbogast and Bedrick (2004) proposed how to check the adequacy of the variance function in a log-linear model. Their methods allow for a large class of smooth alternatives but they have not discussed general heteroscedastic regression models and they assume normal random errors.

In this paper, we present a kernel-smoothing based nonparametric test for assessing the goodness-of-fit of a variance function in a general heteroscedastic regression model. The proposed method does not require to specify a parametric distribution for the random errors and is designed to be powerful against different alternatives. It generalizes the smoothing test of Zheng (1996) for checking the lack-of-fit of the mean function. The next section introduces the test statistic and discusses its asymptotic properties. Section 3 proposes a simple bootstrap algorithm to obtain the critical values for finite sample size. Numerical simulations are reported in Section 4 and the Esterase data from radioimmunoassay study is analyzed in Section 5. Sec- 
tion 6 generalizes the test to the unknown mean function case. Section 7 summarizes the paper. The technical proofs are given in an appendix.

\section{The Testing Procedure}

\subsection{Hypothesis of Interest}

Let $Y$ be a response variable, $X$ be an $l \times 1$ vector of covariates and $Z$ be a $q \times 1$ vector of explanatory variables which may contain part or all components of $X$. A general heteroscedastic regression model based on $n$ independent observation triplets $\left\{\left(X_{i}, Y_{i}, Z_{i}\right), i=1, \ldots, n\right\}$ can be written as

$$
Y_{i}=f\left(X_{i}, \beta\right)+\epsilon_{i}, \quad \sigma_{i}^{2}=g\left(Z_{i}, \beta, \theta\right),
$$

where $f$ is the conditional mean function, $\sigma_{i}^{2}$ denotes the conditional variance function $\operatorname{Var}\left(Y_{i} \mid Z_{i}\right)$, the function $g$ depends on $\beta$ and $\theta$ with the components in $\theta$ distinct from those in $\beta$, and the $\epsilon_{i}$ are independent random errors with mean zero. This formulation includes the popular log-linear model and power-of-the-mean model, where the former has $f\left(X_{i}, \beta\right)=X_{i}^{\prime} \beta, g\left(Z_{i}, \beta, \theta\right)=$ $\exp \left(Z_{i}^{\prime} \beta\right)$ and the latter has $g\left(Z_{i}, \beta, \theta\right)=\theta_{1}\left(f\left(X_{i}, \beta\right)\right)^{\theta}$.

We are interested in testing whether the variance function in (1) can adequately describes the variability in the data. The null hypothesis is

$$
H_{0}: \quad \sigma_{i}^{2}=g\left(Z_{i}, \beta, \theta\right), \quad \text { for } \text { some } \beta, \theta \text {. }
$$

For example, to check the fit of a log-linear structure for the variance function, $H_{0}$ would state that $g$ is an exponential function. The alternative space consists of all twice continuously differentiable functions other than exponential functions.

For the transparency of explaining the main ideas, we assume that the mean function $f$ has a known parametric form in the main body of the paper 
(a way to relax this assumption is given in Section 6). In practical regression analysis, it is rare that a nonparametric model is used to fit the mean but a parametric model is used for the variance. Knowledge of the mean function may come from our understanding of the random mechanism which generates the data, the underlying scientific theory or results from previous or similar studies. We suggest that a goodness-of-fit test for the mean function (the modern smoothing test allows for testing the fit of the mean function without a parametric form for the variance function, see Zheng, 1996) is carried out at the first stage and proceed with a test for the adequacy of the variance function only when the first test does not yield a significant result. In other words, attentions should be first given to the lower-order moment model and then to the higher-order moment model.

\subsection{The Test Statistic}

The test is motivated by the fact $E\left[r_{i} E\left(r_{i} \mid Z_{i}\right) p\left(Z_{i}\right)\right]=E\left[\left(E\left(r_{i} \mid Z_{i}\right)\right)^{2} p\left(Z_{i}\right)\right]$ is zero under $H_{0}$ but is strictly positive for any alternative, where $r_{i}=$ $\epsilon_{i}^{2}-g\left(Z_{i}, \beta, \theta\right)$, and $p(\cdot)$ is the density function of $Z_{i}$.

The test statistic is constructed as an estimator of $E\left[r_{i} E\left(r_{i} \mid Z_{i}\right) p\left(Z_{i}\right)\right]$. First, consider only the outer-layer expectation and estimate this moment by the sample mean $n^{-1} \sum_{i=1}^{n} r_{i} E\left(r_{i} \mid Z_{i}\right) p\left(Z_{i}\right)$. Then, the product $E\left(r_{i} \mid Z_{i}\right) p\left(Z_{i}\right)$ is estimated nonparametrically by

$$
\frac{1}{n-1} \sum_{\substack{j=1 \\ j \neq i}}^{n} \frac{1}{h^{q}} K\left(\frac{Z_{i}-Z_{j}}{h}\right) r_{j}
$$

where $K(\cdot)$ is a kernel function, $h$ is a smoothing parameter which depends on $n$ converges to 0 at an appropriate rate, and $q$ represents the dimension of $Z_{i}$. It is often assumed that $K(u)$ is a nonnegative, bounded, continuous, 
symmetric function and $\int K(u) d u=1$. This estimator is called a "leaveone-out" kernel estimator because the $i$-th observation is left out. Since the $r_{i}$ are not observable, they are replaced by the $\sqrt{n}$-consistent estimators

$$
\widehat{r}_{i}=\left(Y_{i}-f\left(X_{i}, \widehat{\beta}\right)\right)^{2}-g\left(Z_{i}, \widehat{\beta}, \widehat{\theta}\right), \quad i=1, \ldots, n,
$$

where $(\widehat{\beta}, \widehat{\theta})$ is the pseudo-likelihood estimator of $(\beta, \theta)$ (see Section 3.1). The $\widehat{r}_{i}$ 's are correlated due to the estimation of the parameters but we expect them to approximately fluctuate around zero under $H_{0}$. A scatter plot of $\widehat{r}_{i}$ versus $Z_{i}$ (of course, if $Z_{i}$ is univariate), would be a useful graphical display to check the validity of the assumed variance structure.

Assembling the above estimators together, we obtain a kernel-smoothing based nonparametric estimator of $E\left[r_{i} E\left(r_{i} \mid Z_{i}\right) p\left(Z_{i}\right)\right]$, which is given by

$$
T_{n}=\frac{1}{n(n-1)} \sum_{i=1}^{n} \sum_{j \neq i} \frac{1}{h^{q}} K\left(\frac{Z_{i}-Z_{j}}{h}\right) \widehat{r}_{i} \widehat{r}_{j} .
$$

Since large value of $T_{n}$ indicates deviations from the null hypothesis, $T_{n}$ will be used as our test statistic. The statistic $T_{n}$ is a smoothing-based nonparametric estimator of a population moment condition which is zero if and only if the null hypothesis is true, it therefore belongs to the class of so-called "moments tests" which includes many popular testing procedures as special cases such as the Lagrange multiplier test and the information matrix test. Our test statistic should be considered as a generalization of a test proposed by Zheng (1996) for testing the goodness-of-fit of the mean regression function since both tests have similar forms.

Under the null hypothesis, $T_{n}$ can be approximated by

$$
T_{n}^{\prime}=\frac{1}{n(n-1)} \sum_{i=1}^{n} \sum_{j \neq i} \frac{1}{h^{q}} K\left(\frac{Z_{i}-Z_{j}}{h}\right) r_{i} r_{j} .
$$


Note that $T_{n}^{\prime}$ has the same form as $T_{n}$ but with $\widehat{r}_{i}$ replaced by independent quantities $r_{i}$. In fact, if $h \rightarrow 0$ and $n h^{q} \rightarrow \infty$ as $n \rightarrow \infty$, then under smoothness and moment conditions that are similar as in Zheng (1996),

$$
n h^{q / 2}\left(T_{n}-T_{n}^{\prime}\right) \rightarrow 0
$$

in probability under $H_{0}$. The statistic $T_{n}^{\prime}$ has the form of a degenerate secondorder $U$-statistic and the theory developed in Hall (1984) can be applied to derive its asymptotic normality. Under $H_{0}$, we can show that as $n \rightarrow \infty$, $h \rightarrow 0$ and $n h^{q} \rightarrow \infty$,

$$
n h^{q / 2} T_{n}^{\prime} \rightarrow N\left(0, \tau^{2}\right)
$$

in distribution, where $N(a, b)$ denotes the normal distribution with mean $a$ and variance $b$ and

$$
\tau^{2}=2 \int K^{2}(u) d u \int\left[\xi^{4}(z, \beta, \theta)-g^{2}(z, \beta, \theta)\right]^{2} p^{2}(z) d z
$$

with $\xi^{4}(z, \beta, \theta)=E\left(\epsilon_{i}^{4} \mid Z_{i}=z\right)$. Because of (5), the normal distribution given in (6) is also the limiting distribution of $n h^{q / 2} T_{n}$. To test for the adequacy of the specified variance structure, a level $\alpha$ test will reject the null hypothesis if $n h^{q / 2} T_{n} / \tau>\Phi^{-1}(1-\alpha)$, where $\Phi^{-1}(1-\alpha)$ is the $(1-\alpha)$-quantile of the standard normal distribution.

\subsection{Asymptotic Power Properties}

The nonparametric test $T_{n}$ has the property of being consistent for any alternative that is twice continuously differentiable. This omnibus property of $T_{n}$ can be established by showing: for any such alternative, we have $n h^{q / 2} T_{n} \rightarrow \infty$ in probability as $n \rightarrow \infty$. We emphasize that the classical parametric tests are only consistent against certain alternatives. 
Furthermore, the power property is often analyzed for a sequence of local alternatives of the form $\sigma_{i}^{2}=g\left(Z_{i}, \beta, \theta\right)+c_{n} \Delta\left(Z_{i}\right)$, where $c_{n}$ is a sequence of numbers converging to zero, $\Delta\left(Z_{i}\right)$ is a function that is not in the parametric class $\left\{g\left(Z_{i}, \beta, \theta\right): \beta, \theta\right\}$. Of interest is the rate of $c_{n}$ which makes the test have a nontrivial power between zero and one. For parametric tests, the rate is $n^{-1 / 2}$; for smoothing-based nonparametric tests, this rate is generally slower than $n^{-1 / 2}$. We can show that $n h^{q / 2} T_{n}$ has an asymptotic normal distribution with a nonzero mean and the same asymptotic variance as that under the null hypothesis for $c_{n}=O\left(n^{-1 / 2} h^{-q / 4}\right)$. Note that this rate can be made as close as possible to the parametric rate $n^{-1}$ if we let $h$ converge to zero slowly.

\section{Practical Implementation}

\subsection{Pseudo-likelihood Estimation}

The implementation of the test requires estimation of the model under the null hypothesis. The book of Carroll and Ruppert (1988) provides a comprehensive review of methods for fitting heteroscedastic regression models, of which the pseudo-likelihood method has especially been proven to be simple and effective.

Briefly speaking, the pseudo-likelihood procedure involves iterative steps. Given $\beta^{*}$, a current estimator of $\beta$, the estimator of $\theta$ is defined to be the value which maximizes

$$
-\sum_{i=1}^{n} \ln \left(g\left(Z_{i}, \beta^{*}, \theta\right)\right)-\frac{1}{2} \sum_{i=1}^{n} \frac{\left(Y_{i}-f\left(X_{i}, \beta^{*}\right)\right)^{2}}{g\left(Z_{i}, \beta^{*}, \theta\right)} .
$$

Although (7) has the form of a normal likelihood, the pseudo-likelihood makes no assumption about the distribution of the underlying data. Call 
the pseudo-likelihood estimator of $\theta$ obtained at this step $\theta^{*}$, the estimator of $\beta$ is then updated using the generalized least squares method, which is equivalent to solving the equation

$$
\sum_{i=1}^{n} \frac{\partial f\left(X_{i}, \beta\right)}{\partial \beta} \frac{Y_{i}-f\left(X_{i}, \beta\right)}{g\left(Z_{i}, \beta, \theta^{*}\right)}=0 .
$$

Given a starting value of $\beta$, the above process can be repeated until convergence. The estimators obtained are $\sqrt{n}$-consistent under very general conditions.

\subsection{A Bootstrap Algorithm}

It is well known that for nonparametric smoothing tests, the bootstrap procedure usually exhibits better performance for small and moderate sample size, see for example Härdle and Mammen (1993). We state below a simple bootstrap algorithm for the fixed design case. The same algorithm can be slightly modified and applied to the random design setting as well. The bootstrap algorithm consists of the following five steps:

1. For a given random sample of observations, obtain the quasi-likelihood estimator $(\widehat{\beta}, \widehat{\theta})$ of $(\beta, \theta)$ under the null hypothesis.

2. Define $\widehat{\epsilon}_{i}=\left[Y_{i}-f\left(X_{i}, \widehat{\beta}\right)\right] / \sqrt{g\left(Z_{i}, \widehat{\beta}, \widehat{\theta}\right)}, i=1, \ldots, n$. Center and standardize $\widehat{\epsilon}_{1}, \ldots, \widehat{\epsilon}_{n}$ such that they have mean zero and variance one.

3. Obtain a bootstrap sample from the standardized variables obtained in Step 2, call them $\widehat{\epsilon}_{1}^{*}, \ldots, \widehat{\epsilon}_{n}^{*}$, and define $Y_{i}^{*}=f\left(X_{i}, \widehat{\beta}\right)+\sqrt{g\left(Z_{i}, \widehat{\beta}, \widehat{\theta}\right)} \widehat{\epsilon}_{i}^{*}$, $i=1, \ldots, n$.

4. For the bootstrap sample $\left(X_{i}, Y_{i}^{*}, Z_{i}\right), i=1, \ldots, n$, calculate the pseudolikelihood estimator $\left(\widehat{\beta}^{*}, \widehat{\theta}^{*}\right)$ under the null hypothesis, let $\widehat{r}_{i}^{*}=\left(Y_{i}^{*}-\right.$ 
$\left.f\left(X_{i}, \widehat{\beta}^{*}\right)\right)^{2}-g\left(Z_{i}, \widehat{\beta}^{*}, \widehat{\theta}^{*}\right)$. The bootstrap version of the test statistic is

$$
T_{n}^{*}=\frac{1}{n(n-1)} \sum_{i=1}^{n} \sum_{j \neq i} \frac{1}{h^{q}} K\left(\frac{Z_{i}-Z_{j}}{h}\right) \widehat{r}_{i}^{*} \widehat{r}_{j}^{*} .
$$

5. Repeat steps 3 and 4 a large number of times. For a specified nominal level of the test, the critical value is then determined as the appropriate quantile of the bootstrap distribution of the test statistic.

\section{Numerical Simulations}

We investigate the performance of the proposed test in finite sample sizes. The test is calculated with 400 simulation runs and nominal level 0.05. The simulated level thus has a Monte Carlo error of $\sqrt{0.05 * 0.95 / 400} \approx 1 \%$. We use 200 bootstrap samples per run to obtain the critical value. The random data are generated using the statistical software $R$. In the two simulation examples below, we evaluate the goodness-of-fit of the log-linear variance model and the power-of-the-mean model. To investigate the influence of the smoothing parameter, we report the simulation results for different choices of $h$, which reflect different degrees of smoothness.

Simulation study 1: log-linear variance function. For this model, we compare the nonparametric test $T_{n}$ with the classical Wald test which requires to specify an alternative and normal random errors. To test for the log-linear variance structure $\sigma_{i}^{2}=\exp \left(\theta_{0}+\theta_{1} x_{i}\right)$, the Wald test fits a more general variance model $\sigma_{i}^{2}=\exp \left(\theta_{0}+\theta_{1} x_{i}+\theta_{2} x_{i}^{2}\right)$ and evaluates whether the coefficient of the quadratic term $\theta_{2}$ is zero.

We generate $Y_{i}=1+2 x_{i}+\sigma_{i} \epsilon_{i}, i=1, \ldots, n$, where the $x_{i}$ are uniformly distributed on $(0,1)$. The $\epsilon_{i}$ are taken to be independent standard normal random variables in order to make fair comparison with the Wald test. Three 
different functional forms are considered

$$
\begin{aligned}
& \text { (1) } \sigma_{i}=\exp \left(-0.5-0.25 x_{i}\right) \\
& \text { (2) } \sigma_{i}=\exp \left(-0.5-0.25 x_{i}-6\left(x_{i}-0.5\right)^{2}\right) \\
& \text { (3) } \sigma_{i}=\exp \left(-0.5-0.25 x_{i}-1.5\left(\sin \left(2 \pi x_{i}\right)\right)^{2}\right) .
\end{aligned}
$$

Note that functional form (1) corresponds to the null hypothesis.

Table 1 summarizes the proportion of times the null hypothesis is rejected by the two tests for two different sample sizes $n=50$ and $n=100$ and four different choices of the smoothing parameter $h$ : $0.10,0.15,0.20$ and 0.25 .

[Table 1 about here.]

It is observed that the $T_{n}$ test maintains the specified nominal level very well under the null hypothesis while the large-sample Wald-test tends to be somewhat liberal. Our simulation experience indicates that if we raise the sample size to 150, the estimated type I error for the Wald test reduces to 0.063. For the second functional form of $\sigma_{i}$, the Wald test is more powerful than $T_{n}$ for sample size $n=50$ but the power of $T_{n}$ catches up for $n=100$. This is not surprising as this alternative is designed to the advantage of the Wald test. Indeed, the Wald test is most powerful if the true deviation from the log-linear variance structure happens in the log-quadratic direction but it can exhibit inferior power if the deviation happens in other directions. In contrast, the smoothing-based conditional moment test is less powerful than the Wald test when the deviation is in the log-quadratic direction and the random errors are normal, but it can be more powerful than the Wald test for deviations in many other directions. This is demonstrated by the simulation 
results for alternative (3), where the Wald test has very low power while the $T_{n}$ test shows very high power.

Simulation study 2: power-of-the-mean variance function. This model assumes $\sigma_{i}^{2}=\theta_{1}\left(f\left(X_{i}, \beta\right)\right)^{\theta_{2}}$. In theory, if one is willing to assume a parametric error distribution, a parametric test such as likelihood-based test can be constructed. However, this is rarely done in practice because unlike the log-linear variance structure where the log-quadratic variance structure provides a natural extended model, such natural nested structure is not easily available for the power-of-the-mean variance model.

We generate $Y_{i}=20+10 x_{1 i}+10 x_{2 i}+\sigma_{i} \epsilon_{i}, i=1, \ldots, n$, where the $x_{1 i}$ are uniform on $(0,1)$, and the $x_{2 i}$ are uniform on $(-1.5,1.5)$. Three different functional forms are considered for $\sigma_{i}$ :

$$
\begin{aligned}
& \text { (1) } \sigma_{i}=0.05 \mu_{i}^{0.25} \\
& \text { (2) } \sigma_{i}=0.05\left(\mu_{i}^{0.25}+e^{0.08 \mu_{i}}\right), \\
& \text { (3) } \sigma_{i}=0.05\left(\mu_{i}^{0.25}+5 x_{2 i}^{2}\right),
\end{aligned}
$$

where $\mu_{i}=20+10 x_{1 i}+10 x_{2 i}$ is the mean for the $i$-th observation. We also consider three different error distributions for the $\epsilon_{i}$ : standard normal, $t$-distribution with four degrees of freedom, and lognormal. For comparison purpose, the random errors from the $t$-distribution or lognormal distribution are standardized to have mean zero and variance one.

For two different sample sizes $n=50,100$ and four different bandwidths $h=0.10,0.15,0.20$ and 0.25 , the proportion of times the nonparametric test rejects the null hypothesis for various scenarios is summarized in Table 2. The simulation results indicate that the observed level is quite close to 
the specified nominal level 0.05 for different choices of error distributions, bandwidths and sample sizes. The power performance is also satisfactory. The power is higher for normal errors than for the heavier-tailed errors and increases with the sample size.

[Table 2 about here.]

\section{Applications to Esterase Count Data}

For the Esterase count data set discussed in the introduction, Carrol and Ruppert suggest to fit a linear mean regression function. The local linear smoother imposed on the scatter plot in the top panel of Figure 1 indicates overally linearity mean function is a reasonable assumption. We further check the validity of this proposal using the test of Zheng (1996). A plot of the the p-value versus the smoothing parameter $h$ is exhibited as the solid line in the bottom panel of Figure 1. Such a plot is often referred to as a smoothing trace of the test, see for example King, Hart and Wehrly (1991), Young and Bowman (1995). The p-values are high for all choices of $h$. This provides support to the linear mean function.

For most of the immunoassays data analysis in the literature, the variance is assumed to be proportional to the mean, which leads to the following regression model for the esterase data

$$
Y_{i}=\beta_{0}+\beta_{1} x_{i}+\sigma\left(\beta_{0}+\beta_{1} x_{i}\right)^{\theta} \epsilon_{i}, \quad i=1, \ldots, 108,
$$

where the $\epsilon_{i}$ are independent random errors with mean 0 and variance 1 . To test for the adequacy of the power-of-the-mean variance structure, the nonparametric test $T_{n}$ gives p-values much higher than 0.05 for a wide range 
values of $h$, see the dashed line in the bottom panel of Figure 1. The smoothing trace suggests that the $T_{n}$ test provides no evidence against the power-ofthe-mean variance structure. The pseudo-likelihood method gives for model $(12): \widehat{\beta}_{0}=-37.42$ with an estimated standard error $12.11, \widehat{\beta}_{1}=18.16$ with an estimated standard error $0.95, \widehat{\theta}=1.03$ with an estimated standard error 0.10 , and the scale parameter $\sigma$ is estimated to be 0.24 .

In this data set, the response is RIA count. Poisson model is often used for count data, which would correspond to $\theta_{2}=0.5$ in (12). The above estimated model indicates that the esterase data are more heteroscedastic than what a Poisson model would suggest. Merely for comparison purposes, we check the validity of the Poisson variance structure using the $T_{n}$ test. The $T_{n}$ test gives significant p-values for a wide range of $h$. The smoothing trace for testing this hypothesis is plotted as the dotted line in the bottom panel of Figure 1. Thus, the Possion variance structure does not provide an adequate fit for the esterase data.

\section{Unknown Mean Regression Function}

The assumption of a known parametric mean regression function can be relaxed. Consider the following general heteroscedastic regression model:

$$
Y_{i}=m\left(X_{i}\right)+\sigma\left(X_{i}\right) \epsilon_{i}, \quad i=1, \ldots, n,
$$

where $X$ is an $l$-dimensional vector of covariates and the mean function $m(\cdot)$ is only assumed to be smooth, the $\epsilon_{i}$ are independent with mean zero and variance one. We want to test $H_{0}: \sigma^{2}(x)=g(x, \theta)$ for some $\theta$, i.e., whether the variance function $\sigma^{2}(x)$ can be modeled parametrically.

Let $\widehat{m}(x)$ be a kernel-smoothing estimator of $m(x)$. Hall and Carroll 
(1989) verified that the parameter in the parametric variance function can be consistently estimated with $\sqrt{n}$-rate if $m(x)$ is Lipschitz smooth of order $1 / 2$ or more. Denote $\widehat{r}_{i}=\left(Y_{i}-\widehat{m}\left(x_{i}\right)\right)^{2}-g\left(x_{i}, \widehat{\theta}\right)$, where $\widehat{\theta}$ is an estimator of $\theta$. Then the $\widehat{r}_{i}$ estimate $r_{i}=\left(Y_{i}-m\left(x_{i}\right)\right)^{2}-g\left(x_{i}, \theta\right)$, which have mean zero under the null hypothesis. Define the test statistic similarly as before

$$
T_{n}=\frac{1}{n(n-1)} \sum_{i=1}^{n} \sum_{j \neq i} \frac{1}{h^{l}} K\left(\frac{X_{i}-X_{j}}{h}\right) \widehat{r}_{i} \widehat{r}_{j} .
$$

A somewhat more involved proof (sketched in the appendix) shows that under $H_{0}$, as $n \rightarrow \infty, h \rightarrow 0$ and $n h^{l} \rightarrow \infty$,

$$
n h^{l / 2} T_{n} \rightarrow N\left(0, \xi^{2}\right)
$$

in distribution, where $\xi^{2}=2 \int K^{2}(u) d u \int g^{4}(x, \theta)\left(E\left(\epsilon_{i}^{4} \mid x\right)-1\right)^{2} p^{2}(x) d x$.

We explore the finite sample property of the proposed test through a small Monte Carlo study, where the goal is to test whether the variance is homoscedastic, i.e., whether $g$ is a constant function. The random data is simulated from $Y_{i}=0.5+3\left(x_{i}-0.5\right)^{2}+0.25 \epsilon_{i}, i=1, \ldots, n$, where $x_{i}$ is uniformly distributed on $(0,1)$ and the $\epsilon_{i}$ 's are independent standard normal random variables. We compare the test of this section (denoted by $T_{n 1}$ ) with the test in Section 2.2 that assumes a quadratic mean function (denoted by $T_{n 2}$ ) and the test in Section 2.2 with a linear mean function (denoted by $\left.T_{n 3}\right)$. Thus $T_{n 2}$ represents the case in which a correct mean model is used and $T_{n 3}$ uses incorrectly specified mean model. For $T_{n 1}$, a bootstrap procedure similar to that in Section 3.2 is used, where $f\left(X_{i}, \widehat{\beta}\right)$ is replaced by a nonparametric estimator using kernel smoothing with optimal plug-in bandwidth. For three different sample sizes $n=50,100$ and 150, and four 
different bandwidths $h=0.10,0.15,0.20$ and 0.25 , the estimated levels of the three tests are displayed in Table 3 . It is clear that the test $T_{n 3}$ becomes very liberal as the mean function is incorrectly specified. It is also observed that compared with $T_{n 2}$ where the mean function is correctly specified, it takes much large sample size for $T_{n 1}$ to work properly. Thus the test with unknown mean is not as efficient as the test with a correctly specified parametric mean function, on the other hand, a test with an incorrectly specified parametric mean function may seriously impair the test for the variance function.

[Table 3 about here.]

\section{Summary}

We have developed a nonparametric test for assessing the adequacy of an assumed variance structure in a linear/nonlinear heteroscedastic regression model. The emphasis of this paper is the case the mean function has a known parametric form. This is motivated by the fact that in practice when a parametric form is assumed for a higher moment (the variance), a parametric form is almost always assumed for the lower moment (the mean). We have also discussed a generalization where the mean function is only assumed to be smooth and estimated nonparametrically, but its practical performance needs further study.

\section{ACKNOWLEDGEMENTS}

We would like to thank the $\mathrm{AE}$, a referee and the Co-editor, whose comments led to significant improvement of the paper. This research was funded in part by AHRQ grant R01HS013105 and U.S. Department of Veterans Affairs Health Administration, HSR\&D grant ECI-03-206. 


\section{References}

[1] Arbogast, P. G. and Bedrick, E. J. (2004). Model-checking techniques for linear models with parametric variance functions. Technometrics. 46, 404-410.

[2] Bedrick, E. J. (2000). Checking for lack of fit in linear models with parametric variance functions Technometrics. 42, 227-236.

[3] Breusch, T. S. and Pagan, A. R. (1980). A simple test for heteroscedasticity and random coefficient variation. Econometrica 47, 1287-1294.

[4] Cai, Z. W. Hurvich, C. M. and Tsai, C-L (1998). Score Tests for Heteroscedasticity in Wavelet Regression. Biometrika. 85, 229-234.

[5] Carroll, R. and Ruppert, D (1988). Transformation and weighting in regression. New York: Chapman \& Hall.

[6] Cook, D. and Weisberg, S (1983). Diagnostics for heteroscedasticity in regression. Biometrika. 70, 1-10.

[7] Diblasi, A. and Bowman, A. (1997). Testing for a constant variance in a linear model. Statistics $\&$ Probability Letters, 33, 95-103.

[8] Davidian, M. Carroll, R. and Smith, W. (1988). Variance functions and the minimum detectable concentration in assays. Biometrika. 75, 549556.

[9] Hall, P. (1984), Central limit theorem for integrated square error of multivariate nonparametric density estimators. Journal of Multivariate Analysis, 14, 1-16. 
[10] Hall, P. and Carroll, R. J. (1989), Variance function estimation in regression: The effect of estimating the mean. Journal of the Royal Statistical Society, Series B, 51, 3-14.

[11] Härdle, W., and Mammen, E. (1993), Comparing nonparametric versus parametric regression fits. The Annals of Statistics, 21, 1926-1947.

[12] King, E., Hart, J. D. and Wehrly, T. E. (1991). Testing the equality of two regression curves using linear smoothers. Statistics and Probability Letters, 12, 239-247.

[13] Müller, H. G. and Zhao, P. L. (1995). On a semiparametric variance model and a test for heteroscedasticity. Ann. Statist. 23, 946-967.

[14] Ruppert, D., Wand, M. P., Holst, U., and Hössjer, O. (1997), "Local polynomial variance-function estimation", Technometrics, 39, 262-273.

[15] Stute, W. (1984). The oscillation behavior of empirical processes: the multivariate case. The Annals of Probability. 12, 361-379.

[16] White, H. (1980). A heteroscedasticity-consistent covariance matrix estimator and a direct test for heteroscedasticity. Econometrica. 48, 817838.

[17] Young, S. G. and Bowman, A. W. (1995). Non-parametric analysis of covariance. Biometrics, 51, 920-931.

[18] Zheng, J. X. (1996), A consistent test of functional form via nonparametric estimation techinques. Journal of Econometrics, 75, 263-289. 
[19] Zhou, X.H. , Stroupe, K.T. and Tierney, W.M. (2001). Regression analysis of health care charges with heteroscedasticity. JRSS, Ser. C. 50(3), 303-312.

\section{APPENDiX A}

Sketch of Proofs

Proof of (5). Since $\widehat{r}_{i}=\left(Y_{i}-f\left(X_{i}, \widehat{\beta}\right)\right)^{2}-g\left(Z_{i}, \widehat{\beta}, \widehat{\theta}\right), r_{i}=\left(Y_{i}-f\left(X_{i}, \beta\right)\right)^{2}-$ $g\left(Z_{i}, \beta, \theta\right)$, we have $\widehat{r}_{i}=r_{i}+2 \epsilon_{i}\left(f\left(X_{i}, \beta\right)-f\left(X_{i}, \widehat{\beta}\right)\right)+\left(f\left(X_{i}, \beta\right)-f\left(X_{i}, \widehat{\beta}\right)\right)^{2}+$ $\left(g\left(Z_{i}, \beta, \theta\right)-g\left(Z_{i}, \widehat{\beta}, \widehat{\theta}\right)\right)$. As a result, $T_{n}$ can be decomposed as a sum of ten terms: $T_{n}=T_{n}^{\prime}+\sum_{i=1}^{9} Q_{i}$, where

$$
\begin{aligned}
Q_{1} & =\frac{4}{n(n-1) h^{q}} \sum_{i=1}^{n} \sum_{j=1, j \neq i}^{n} K\left(\frac{Z_{i}-Z_{j}}{h}\right) r_{i} \epsilon_{j}\left(f\left(X_{j}, \beta\right)-f\left(X_{j}, \widehat{\beta}\right)\right), \\
Q_{2} & =\frac{2}{n(n-1) h^{q}} \sum_{i=1}^{n} \sum_{j=1, j \neq i}^{n} K\left(\frac{Z_{i}-Z_{j}}{h}\right) r_{i}\left(f\left(X_{j}, \beta\right)-f\left(X_{j}, \widehat{\beta}\right)\right)^{2},
\end{aligned}
$$

and $Q_{i}, i=3, \ldots, 9$, are similarly defined. Let $\frac{\partial f\left(X_{j}, \beta\right)}{\partial \beta}$ be the $m \times 1$ vector with the $i$ th element $\frac{\partial f\left(X_{j}, \beta\right)}{\partial \beta_{i}}$, and $\frac{\partial f\left(X_{j}, \beta\right)}{\partial \beta^{\prime}}$ be the transpose of this vector. Let $\frac{\partial^{2} f\left(X_{j}, \beta\right)}{\partial \beta \partial \beta^{\prime}}$ be an $m \times m$ matrix with the $(i, k)$ th element $\frac{\partial^{2} f\left(X_{j}, \beta\right)}{\partial \beta_{i} \partial \beta_{k}}$, then we have

$$
\begin{aligned}
Q_{1}= & \frac{4}{n(n-1) h^{q}} \sum_{i=1}^{n} \sum_{j=1, j \neq i}^{n} K\left(\frac{Z_{i}-Z_{j}}{h}\right) r_{i} \epsilon_{j} \frac{\partial f\left(X_{j}, \beta\right)}{\partial \beta^{\prime}}(\beta-\widehat{\beta}) \\
& +(\beta-\widehat{\beta})^{\prime} \frac{4}{n(n-1) h^{q}} \sum_{i=1}^{n} \sum_{j=1, j \neq i}^{n} K\left(\frac{Z_{i}-Z_{j}}{h}\right) r_{i} \epsilon_{j} \frac{\partial^{2} f\left(X_{j}, \bar{\beta}\right)}{\partial \beta \partial \beta^{\prime}}(\beta-\widehat{\beta}) \\
= & Q_{11}(\beta-\widehat{\beta})+(\beta-\widehat{\beta})^{\prime} Q_{12}(\beta-\widehat{\beta}),
\end{aligned}
$$

where the definition of $Q_{11}$ and $Q_{12}$ should be clear from the context, $\bar{\beta}$ depends on $X_{j}$ and lies between $\beta$ and $\widehat{\beta}$. Note that the $r_{i}$ 's are independent 
with mean 0, thus $E\left(Q_{11}\right)=0$ and

$$
\begin{aligned}
& E\left(Q_{11}^{2} \mid X, Z\right) \\
& =\frac{16}{n^{2}(n-1)^{2} h^{2 q}} \sum_{i_{1}=1}^{n} \sum_{i_{2}=1}^{n} \sum_{j_{1}=1, j_{1} \neq i_{1}}^{n} \sum_{j_{2}=1, j_{2} \neq i_{2}}^{n} K\left(\frac{Z_{i_{1}}-Z_{j_{1}}}{h}\right) K\left(\frac{Z_{i_{2}}-Z_{j_{2}}}{h}\right) \\
& E\left(r_{i_{1}} r_{i_{2}} \epsilon_{j_{1}} \epsilon_{j_{2}}\right) \frac{\partial f\left(X_{j_{1}}, \beta\right)}{\partial \beta^{\prime}} \frac{\partial f\left(X_{j_{2}}, \beta\right)}{\partial \beta^{\prime}} .
\end{aligned}
$$

In order for the expectation to be nonzero, we must have $i_{1}=i_{2}$ and $j_{1}=$ $j_{2}$ or $i_{1}=j_{2}$ and $i_{2}=j_{1}$, we have $E\left(Q_{11}^{2} \mid X, Z\right)=O\left(n^{-4} h^{-2 q}\right) O\left(n^{2}\right)=$ $O\left(n^{-2} h^{-2 q}\right)$. Since the quasi-likelihood estimator $\widehat{\beta}$ is $\sqrt{n}$-consistent for $\beta$, we have $n h^{q / 2} Q_{11}(\beta-\widehat{\beta})=O\left(n h^{q / 2}\right) O_{p}\left(n^{-1} h^{-q}\right) O_{p}\left(n^{-1 / 2}\right)=O_{p}\left(n^{-1 / 2} h^{-q / 2}\right)=$ $o_{p}(1)$. Similarly, $Q_{12}=O_{p}(1)$ and $n h^{q / 2}(\beta-\widehat{\beta})^{\prime} Q_{12}(\beta-\widehat{\beta})=O_{p}\left(h^{q / 2}\right)=$ $o_{p}(1)$. Therefore $n h^{q / 2} Q_{1}=o_{p}(1)$. Similarly, we can show $n h^{q / 2} Q_{i}=o_{p}(1)$, $i=2, \ldots, 9$.

Proof of (6). From (5), $n h^{q / 2} T_{n}$ and $n h^{q / 2} T_{n}^{\prime}$ have the same asymptotic distribution. Since the $r_{i}$ 's are independent with mean $0, n h^{q / 2} T_{n}^{\prime}$ is a secondorder degenerate $U$-statistic. Its asymptotic normality can be established by checking the condition of Theorem 1 of Hall (1984).

Proof of (15). For $\widehat{r}_{i}=\left(Y_{i}-\widehat{m}\left(X_{i}\right)\right)^{2}-g\left(X_{i}, \widehat{\theta}\right)$, where $\widehat{m}\left(X_{i}\right)=[(n-$ 1) $\left.h^{l}\right]^{-1} \sum_{k \neq i} Y_{k} K\left(\left(X_{k}-X_{i}\right) / h\right) / \widehat{p}\left(X_{i}\right)$ and $\widehat{p}\left(X_{i}\right)=\left[(n-1) h^{l}\right]^{-1} \sum_{k \neq i} K\left(\left(X_{k}-\right.\right.$ $\left.\left.X_{i}\right) / h\right)$, and $r_{i}=\left(Y_{i}-m\left(X_{i}\right)\right)^{2}-g\left(X_{i}, \theta\right)$, we have $\widehat{r}_{i}=r_{i}+2 \sigma\left(X_{i}\right) \epsilon_{i}\left(m\left(X_{i}\right)-\right.$ $\left.\widehat{m}\left(x_{i}\right)\right)+\left(m\left(X_{i}\right)-\widehat{m}\left(X_{i}\right)\right)^{2}+\left[g\left(X_{i}, \theta\right)-g\left(X_{i}, \widehat{\theta}\right)\right]$. Similarly as in the proof of (5), $T_{n}$ can be decomposed as a sum of ten terms: $T_{n}=T_{n}^{\prime}+\sum_{i=1}^{9} Q_{i}$, where $T_{n}^{\prime}=\left[n(n-1) h^{l}\right]^{-1} \sum_{i=1}^{n} \sum_{j \neq i} K\left(\frac{X_{i}-X_{j}}{h}\right) r_{i} r_{j}$

$$
Q_{1}=\frac{4}{n(n-1) h^{l}} \sum_{i=1}^{n} \sum_{j=1, j \neq i}^{n} K\left(\frac{X_{i}-X_{j}}{h}\right) r_{i} \sigma\left(X_{j}\right) \epsilon_{j}\left(m\left(X_{j}\right)-\widehat{m}\left(X_{j}\right)\right),
$$




$$
Q_{2}=\frac{2}{n(n-1) h^{l}} \sum_{i=1}^{n} \sum_{j=1, j \neq i}^{n} K\left(\frac{X_{i}-X_{j}}{h}\right) r_{i}\left(m\left(X_{j}\right)-\widehat{m}\left(X_{j}\right)\right)^{2},
$$

and $Q_{i}, i=3, \ldots, 9$, are similarly defined. To show $n h^{l / 2} Q_{1}=o_{p}(1)$, we make use of the following fact:

$$
\begin{aligned}
m\left(X_{j}\right)-\widehat{m}\left(X_{j}\right)= & \frac{\widehat{s}\left(X_{j}\right)-s\left(X_{j}\right)}{p\left(X_{j}\right)}-\frac{\left(\widehat{s}\left(X_{j}\right)-s\left(X_{j}\right)\right)\left(\widehat{p}\left(X_{j}\right)-p\left(X_{j}\right)\right)}{p\left(X_{j}\right) \widehat{p}\left(X_{j}\right)} \\
& -\frac{s\left(X_{j}\right)\left(\widehat{p}\left(X_{j}\right)-p\left(X_{j}\right)\right)}{p^{2}\left(X_{j}\right)}+\frac{s\left(X_{j}\right)\left(\widehat{p}\left(X_{j}\right)-p\left(X_{j}\right)\right)^{2}}{p^{2}\left(X_{j}\right) \widehat{p}^{2}\left(X_{j}\right)},
\end{aligned}
$$

where $s\left(X_{j}\right)=m\left(X_{j}\right) p\left(X_{j}\right)$ and $\widehat{s}\left(X_{j}\right)=\widehat{m}\left(X_{j}\right) \widehat{p}\left(X_{j}\right)$. Based on the above decomposition, $n h^{l / 2} Q_{1}$ can be written as $n h^{l / 2} Q_{1}=Q_{11}+Q_{12}+Q_{13}+Q_{14}$.

For instance,

$$
Q_{11}=\frac{4}{n(n-1) h^{l}} \sum_{i=1}^{n} \sum_{j=1, j \neq i}^{n} K\left(\frac{X_{i}-X_{j}}{h}\right) r_{i} \sigma\left(X_{j}\right) \epsilon_{j} \frac{\widehat{s}\left(X_{j}\right)-s\left(X_{j}\right)}{p\left(X_{j}\right)} .
$$

Since

$$
\begin{aligned}
& \widehat{s}\left(X_{j}\right)-s\left(X_{j}\right) \\
= & \frac{1}{(n-1) h^{l}} \sum_{k \neq j} K\left(\frac{X_{i}-X_{j}}{h}\right)\left(m\left(X_{k}\right)-m\left(X_{j}\right)\right) \\
& +\frac{1}{(n-1) h^{l}} \sum_{k \neq j} K\left(\frac{X_{i}-X_{j}}{h}\right) \sigma\left(X_{k}\right) \epsilon_{k}+m\left(X_{j}\right)\left(\widehat{p}\left(X_{j}\right)-p\left(X_{j}\right)\right),
\end{aligned}
$$

$Q_{11}$ can be further written as $Q_{11}=Q_{11 A}+Q_{11 B}+Q_{11 C}$. By directly checking mean and variance, we can show $Q_{11 A}=o_{p}(1), Q_{11 B}=o_{p}(1)$. And we can show $Q_{11 C}=o_{p}(1)$ by employing a result of Stute (1984): $\sup _{x}|\widehat{p}(x)-p(x)|=$ $\left(n^{-1} h^{-l}\left(\ln h^{-l}\right)\right)^{1 / 2}$ almost surely. This proves that $Q_{11}=o_{p}(1)$. Similarly, we can show $Q_{1 i}=o_{p}(1)$, for $i=2,3$, 4 , which yields $Q_{1}=o_{p}(1)$. We prove $n h^{l / 2}\left(T_{n}-T_{n}^{\prime}\right)=o_{p}(1)$ by showing $Q_{i}=o_{p}(1)$, for $i=2, \ldots, 9$ using the same technique. The asymptotic normality is proved by applying the result of Hall (1984) on $T_{n}^{\prime}$. 

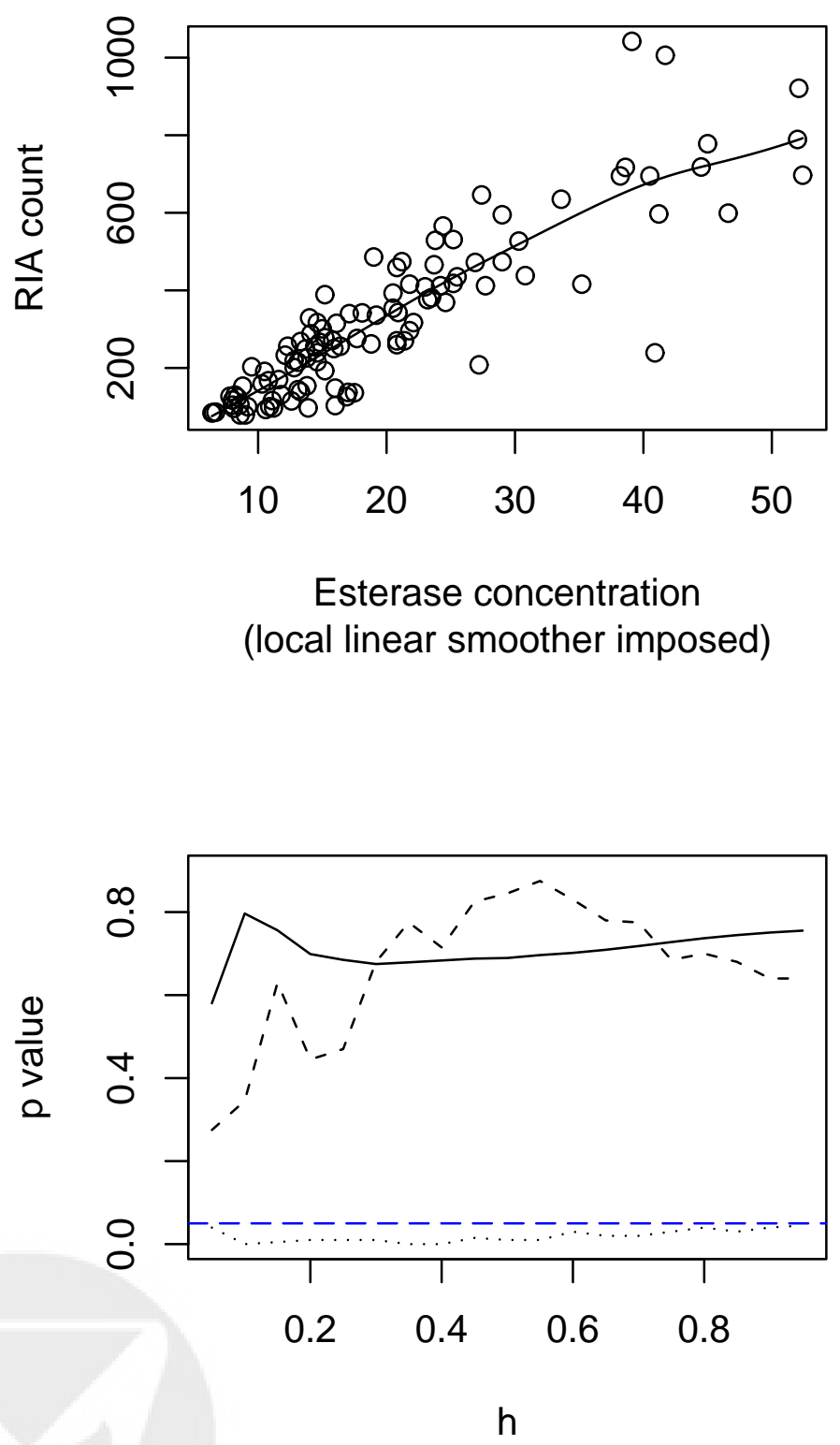

Figure 1. Analysis of Esterase data. The top graph is a scatter plot; the bottom graph contains smoothing traces for three different hypotheses: The solid line is for testing the linearity of the mean function; the dashed line is for testing the power-of-the-mean variance structure, the dotted line is for testing Poisson model, and the horizontal dashed line has intercept 0.05. 
Table 1

Estimated powers of the $T_{n}$ test and the Wald test for the three functional forms of $\sigma\left(x_{i}\right)$ specified in (10) and two different sample sizes $n=50,100$. The nominal level is 0.05 .

\begin{tabular}{|c|c|c|c|c|c|}
\hline \multirow[b]{2}{*}{$\sigma\left(x_{i}\right)$} & \multirow[b]{2}{*}{$h$} & \multicolumn{2}{|c|}{$n=50$} & \multicolumn{2}{|c|}{$n=100$} \\
\hline & & $T_{n}$ test & Wald test & $T_{n}$ test & Wald test \\
\hline \multirow{4}{*}{ (1) } & 0.10 & 0.048 & 0.103 & 0.055 & 0.070 \\
\hline & 0.15 & 0.050 & & 0.053 & \\
\hline & 0.20 & 0.048 & & 0.053 & \\
\hline & 0.25 & 0.050 & & 0.053 & \\
\hline \multirow{4}{*}{$(2)$} & 0.10 & 0.633 & 0.995 & 0.943 & 1.000 \\
\hline & 0.15 & 0.735 & & 0.970 & \\
\hline & 0.20 & 0.780 & & 0.983 & \\
\hline & 0.25 & 0.810 & & 0.988 & \\
\hline \multirow{4}{*}{ (3) } & 0.10 & 0.658 & 0.140 & 0.973 & 0.165 \\
\hline & 0.15 & 0.690 & & 0.980 & \\
\hline & 0.20 & 0.648 & & 0.963 & \\
\hline & 0.25 & 0.513 & & 0.903 & \\
\hline
\end{tabular}




\section{Table 2}

Estimated powers of the $T_{n}$ test for the three functional forms of $\sigma\left(x_{i}\right)$ specified in (11), three different error distributions and two different sample sizes $n=50,100$. The nominal level is 0.05.

\begin{tabular}{|c|c|c|c|c|c|c|c|}
\hline \multirow[b]{2}{*}{$\sigma\left(x_{i}\right)$} & \multirow[b]{2}{*}{$h$} & \multicolumn{3}{|c|}{$n=50$} & \multicolumn{3}{|c|}{$n=100$} \\
\hline & & normal & $t_{4}$ & lognormal & normal & $t_{4}$ & lognormal \\
\hline \multirow{4}{*}{ (1) } & 0.10 & 0.053 & 0.058 & 0.045 & 0.063 & 0.063 & 0.045 \\
\hline & 0.15 & 0.043 & 0.050 & 0.050 & 0.043 & 0.058 & 0.025 \\
\hline & 0.20 & 0.038 & 0.050 & 0.040 & 0.040 & 0.050 & 0.048 \\
\hline & 0.25 & 0.053 & 0.043 & 0.045 & 0.030 & 0.040 & 0.045 \\
\hline \multirow{4}{*}{ (2) } & 0.10 & 0.455 & 0.305 & 0.180 & 0.773 & 0.473 & 0.238 \\
\hline & 0.15 & 0.533 & 0.350 & 0.183 & 0.848 & 0.550 & 0.230 \\
\hline & 0.20 & 0.598 & 0.383 & 0.190 & 0.885 & 0.608 & 0.268 \\
\hline & 0.25 & 0.665 & 0.418 & 0.193 & 0.933 & 0.645 & 0.248 \\
\hline \multirow{4}{*}{ (3) } & 0.10 & 0.583 & 0.370 & 0.260 & 0.875 & 0.610 & 0.313 \\
\hline & 0.15 & 0.708 & 0.468 & 0.323 & 0.945 & 0.728 & 0.398 \\
\hline & 0.20 & 0.750 & 0.505 & 0.343 & 0.968 & 0.790 & 0.443 \\
\hline & 0.25 & 0.765 & 0.545 & 0.335 & 0.990 & 0.792 & 0.463 \\
\hline
\end{tabular}




\section{Table 3}

Estimated levels of three tests for testing homoscedasticity when the mean function is quadratic. $T_{n 1}$ assumes unknown mean function and estimates it nonparametrically; $T_{n 2}$ assumes a quadratic mean function and $T_{n 3}$ assumes a linear mean function. The nominal level is 0.05.

\begin{tabular}{cccccc}
\hline \hline & & \multicolumn{3}{c}{ test } \\
\cline { 3 - 6 } sample size & $h$ & $T_{n 1}$ & $T_{n 2}$ & $T_{n 3}$ \\
\cline { 3 - 6 } & \multirow{2}{*}{50} & 0.10 & 0.123 & 0.065 & 0.175 \\
& 0.15 & 0.088 & 0.063 & 0.110 \\
& 0.20 & 0.080 & 0.060 & 0.068 \\
& 0.25 & 0.080 & 0.043 & 0.030 \\
\hline \multirow{3}{*}{100} & 0.10 & 0.080 & 0.073 & 0.530 \\
& 0.15 & 0.075 & 0.065 & 0.478 \\
& 0.20 & 0.075 & 0.050 & 0.330 \\
& 0.25 & 0.075 & 0.070 & 0.228 \\
\hline \multirow{3}{*}{150} & 0.10 & 0.055 & 0.048 & 0.813 \\
& 0.15 & 0.058 & 0.048 & 0.780 \\
& 0.20 & 0.050 & 0.045 & 0.635 \\
& 0.25 & 0.050 & 0.048 & 0.430 \\
\hline \hline
\end{tabular}

